\title{
Burning issues in blood pressure control
}

\section{JA Ker, ${ }^{1}$ K Outhoff ${ }^{2}$}

'Department of Internal Medicine, School of Medicine, Faculty of Health Sciences, University of Pretoria, South Africa 2Department of Pharmacology, School of Medicine, Faculty of Health Sciences, University of Pretoria, South Africa Corresponding author, email: kim.outhoff@up.ac.za

Globally more than 1 billion people have hypertension and it is predicted that because of ageing populations and increasing sedentary lifestyles, this figure will rise to about 1.5 billion by 2025 . Elevated blood pressure (BP) is the leading cause of premature death and morbidity due to stroke and ischemic heart disease. Hypertension is also a major risk factor for heart failure, atrial fibrillation, chronic kidney disease, peripheral arterial disease and cognitive decline. This article discusses the current evidence-based treatment guidelines and pressing temporal issues in optimal blood pressure control.

Keywords: blood pressure, hypertension, single pill combination, early blood pressure control, treatment persistence, legacy effects of rapid control

Republished with permission from South African Family Practice 2019; 61(2):24-26

\section{Background}

The recent joint European Societies of Cardiology and Hypertension (ESC/ESH) guideline on the treatment of adult hypertension recommends initiating drug therapy at a blood pressure of $>140 / 90 \mathrm{mmHg}$ after 3-6 months of lifestyle management that fails. ${ }^{1}$ Although there is a continuous relationship between BP and cardiovascular and renal events, considerable evidence suggests that the benefits unequivocally outweigh the risks of treatment at this BP cut-off point, unless the patient is a high risk, in which case drug treatment would be initiated earlier. What is really new in this Guideline is the recommendation to start with one pill, specifically a single pill combination of two drugs, and preferably a renin-angiotensin system (RAS)-blocker combined with either a diuretic or a calcium-channel blocker (CCB). The reason for this is that the single pill combination will improve the speed of BP lowering, improve the efficiency of BP lowering, improve the metabolic side effect profile of single drug therapy, improve adherence to prescribed therapy and ultimately improve BP control. The only exceptions to this initial two-drug combination strategy are low-risk patients with stage 1 hypertension with a SBP $<150 \mathrm{mmHg}$, high-risk patients with high-normal $\mathrm{BP}$, and frail elderly patients, where initiating monotherapy is preferred. The first blood pressure treatment goal is $<140 / 90 \mathrm{mmHg}$ for all, and then to consider a goal of $120-130 / 80 \mathrm{mmHg}$ in those able to tolerate it. In elderly patients (older than 65 years) a higher SBP target of $130-140 \mathrm{mmHg}$ should be aimed for. The recommendation is to reach target BP within 3 months. Despite all these recommendations many people are unaware that they have hypertension, or they are untreated or do not receive therapy that has the proven ability to reduce blood pressure and reduce the adverse outcomes associated with hypertension.

An important question is which could be more important: To reach a lower blood pressure goal as is now recommended or to reach the $\mathrm{BP}<140 / 90 \mathrm{mmHg}$ goal as early as possible? This question has never been evaluated in a randomised clinical trial but the results of observational data seem to indicate that the best preventative effect is achieved with early and sustained controlled blood pressure. ${ }^{2}$

\section{Early blood pressure control}

There are many reasons for the well documented poor control of hypertension globally, and among the possible reasons, late or ineffective treatment leading to irreversible or difficult to reverse adaptations of the cardiovascular system could play a significant role.

An observational 1:1 matched cohort study from the USA and Canada evaluated the role of initial combination therapy versus initial monotherapy and subsequent switching to combination therapy. Conceptually, this evaluated early effective BP control versus staged and later optimal BP control. ${ }^{3}$ After 6 months, $40.3 \%$ and $32.6 \%$ of patients on initial versus delayed combination therapy, reached target BP control respectively. There was a significant reduction of cardiovascular (CV) events in the group who received the initial combination therapy with a hazard ratio of CV events and death of 0.66 ( $95 \% \mathrm{Cl}: 0.52-0.84), p=0.0008$. Initial combination therapy was associated with rapid achievement of BP control. Although some studies hint at the concept of rapid blood pressure control, its precise definition, its significance, and which therapeutic strategy should be chosen to enable rapid control are still lacking. There is no firm recommendation on the diagnostic-to-control time in hypertension, although the European Guidelines on Hypertension do mention 3-6 months to reach control.

In the Systolic Hypertension in Europe (SYST-EUR) trial, where active treatment was tested against placebo in 4695 hypertensive patients, the trial was terminated after 2 years due to significant benefit in the treatment arm. Once the trial was stopped, the 
same combination antihypertensive therapy was given to the placebo group who did not initially receive drug therapy, and this enabled an interesting comparison of early with late onset or delayed therapy. ${ }^{4}$ There were significantly improved outcomes in the early group when followed up for an additional 4.1 years, including reduction of the event rate of stroke from 10.1/1 000 to 7.3/1 000 patient-years [RRR 28\% (95\% Cl: 7-44\%); $p<.01]$.

The VALUE trial compared valsartan combined with hydrochlorothiazide (HCTZ) to amlodipine combined with HCTZ. Within 1 month, systolic blood pressure decreased by $4.0 \mathrm{mmHg}$ more in the amlodipine arm than in the valsartan arm and this led to significant early reductions of myocardial infarction in the amlodipine arm. It was shown that it was this early (within 6 months) reduction of SBP that was responsible for the benefit; later in the trial, when the differences in blood pressure in the two arms were small, this difference in outcome disappeared and became non-significant. Even better results were seen in the early responders if their SBP was reduced by $10 \mathrm{mmHg}$ within the first month. ${ }^{5}$ These results led to the concept that early blood pressure responders benefit more than late responders.

A "real world" study tested the prognostic value of early control of blood pressure compared to delay in control in 18721 newly diagnosed hypertensive patients who were followed up for an average of 5.4 years. $^{6}$ The mean Diagnosis-Control Time (D-C Time) was 49 days. The D-C times were divided into $<29$ days, 30-124 days, and $>125$ days and the outcomes were measured between the different categories. As D-C time became longer, the mortality became higher, with an absolute reduction of mortality of at least $2 \%$ between the early $(<29)$ and later (> 125) D-C days, translating into numbers-needed-to-treat (NNT) of 50 over a treatment period of 5.4 years. Independent predictors of long D-C times were higher initial BP levels, obesity, diabetes mellitus and male gender.

Another important concept, that of time-spent-in-control, was tested in a large observational study in 150130 newly diagnosed hypertensive patients (Unpublished data from the English TITRE trial).? Time-in-control of less than 3 months had a worse outcome than a longer time-in-control.

\section{Persistence of prescribed treatment}

A "real world" Australian study using data from $10 \%$ of the population as a random sample, compared an initial single pill combination of amlodipine plus perindopril versus the two individual drugs not in single pill combination. The aim was to test persistence with the prescribed treatment strategies and to evaluate associated mortality. ${ }^{8}$ The median persistence time for the single combination pill was 42 months $(95 \% \mathrm{Cl}$ : 33-43 months) compared to 7 months when the same two pills were taken separately. The mortality was $8 \%$ with the single pill compared to $18 \%$ when the same two drugs were taken separately. Adherence to antihypertensive medication is thus critical for improved outcomes. This was underscored by a large observational study of 242594 hypertensive patients in one Italian region, which demonstrated that when followed-up for 6 years, those patients who did not discontinue treatment had a relative risk reduction of $37 \%$ in cardiovascular outcomes compared to those who had one episode of discontinuation. ${ }^{9}$

\section{Long-term effect of initial fast control}

A meta-analysis of ten blood pressure lowering trials involving 48 892 patients examined the long-term effects of blood pressure lowering on mortality after trials were terminated..$^{10}$ The results showed a mean in-trial time of 4 years and mean post-trial period of observation of 6 years. There were significant benefits with reduction of in-trial mortality, which although attenuated posttrial, remained significant. This result indicated a persistence of benefit and the importance of continued hypertension treatment.

The question is if there could be a legacy of benefit when hypertensive patients are treated initially with an effective drug combination. This was evaluated in the recently published ASCOTLEGACY study in which the long-term effects of early control with a specific combination were evaluated for a total of 16 years of which 10 years were post-trial. ${ }^{11}$ ASCOT-LEGACY followed up the UK group of 8580 patients who participated in the original ASCOT-BPLA. The ASCOT-BPLA trial tested an amlodipine-based combination (with perindopril, a long-acting ACE-inhibitor) in 19257 patients (from UK, Ireland, Nordic countries) with hypertension and at least 3 other cardiovascular risk factors. ${ }^{12}$ This study demonstrated statistically significant reductions in total mortality, cardiovascular mortality and total stroke, with an absolute risk reduction of $1 \%$ and number-needed-to-treat (NNT) of 100 over 5.5 years in favour of the amlodipine-based regimen. In the ASCOT-LEGACY trial, with a mean total followup of 15.5 years ( 5.5 years in-trial and more than 10 years posttrial), all-cause mortality was not significantly different between the 2 groups post-trial, but stroke mortality was statistically significantly reduced in the amlodipine-perindopril arm with a relative risk reduction (RRR) of $29 \%(95 \% \mathrm{Cl}: 3-47 \% ; p=0.03)$, absolute risk reduction of $0.6 \%$ and NNT of 167 . In the non-LLA of ASCOT, the amlodipine-perindopril reduced coronary mortality with RRR of $21 \%$ (95\% Cl: 7-33\%; $p=0.0046$ ) and absolute risk reduction of $1.2 \%$ with a NNT of 83 over 15.5 years. Coronary artery disease was reduced with a RRR $24 \%$ (95\% Cl: 1-41\%; $p$ $=0.039$ ) and absolute risk reduction of $0.5 \%$ with a NNT of 200 over 15.5 years. In the statin arm of the ASCOT-LLA, long-term cardiovascular mortality was reduced with a RRR of $15 \%$ (95\% $\mathrm{Cl}: 1-28 \% ; p=0.039$ ) and absolute risk reduction of $1.9 \%$ and NNT of 53 over 15.5 years. The ASCOT-LEGACY trial is the first to evaluate both blood pressure lowering and statin effects in the longest trial follow-up (about 16 years). The suggestion from this trial is that there may be a legacy effect from early effective combination therapy using a proven combination of drugs. It also emphasises the importance of the long-term benefits of treating hypertension.

In summary, to effectively minimise the adverse clinical outcomes of hypertension, reducing blood pressure to low levels such as SBP $<130 \mathrm{mmHg}$ is important. Analyses of emerging post-hoc and observational data show that early rapid control that is sustained over time is equally important. A short time to 
BP control significantly benefits patients with hypertension, and most data demonstrate an optimal 3-6 month period to reach control targets. Persistence with prescribed evidence-based drugs for the treatment of hypertension is associated with lower mortality in real-life. Long-term benefits of the calcium channel blocker-based treatment, which seems to be independent of mean blood pressure levels reported in the original ASCOT trial, suggest additional mechanisms for long-term protection. Could the early effective blood pressure reduction of the combination of drugs have played a role in this possible legacy effect? It also appears that blood pressure and cholesterol interventions are associated with long-term benefits in terms of cardiovascular outcomes.

\section{References}

1. Williams B, Mancia G, Spiering W, et al. 2018 Practice guidelines for the management of arterial hypertension of the European Society of Hypertension and the European Society of Cardiology. J Hypertension. 2018;36:2284-309. https://doi.org/10.1097/HJH.0000000000001961.

2. Mariampillai JE, Eskas PA, Heimark S, et al. A case for less intensive blood pressure control: It matters to achieve target blood pressure early and sustained below 140/90 mmHg. Progress in Cardiovasc Dis. 2016;59:209-18. https://doi. org/10.1016/j.pcad.2016.09.002.

3. Gradman AH, Parisé $H$, Lefebvre $P$, et al. Initial combination therapy reduces the risk of cardiovascular events in hypertensive patients: A matched cohort study. Hypertension. 2013;61:309-18. https://doi.org/10.1161/ HYPERTENSIONAHA.112.201566.

4. Staessen JA, Thijs L, Fagard R, et al. Effects of immediate versus delayed anti-hypertensive therapy on outcome in the Systolic Hypertension
Trial in Europe (SYST-EUR). J Hypertension. 2004;22:847-57. https://doi. org/10.1097/00004872-200404000-00029.

5. Weber MA, Julius S, Kjeldsen SE, et al. Blood pressure dependent and independent effects of antihypertensive treatment on clinical events in the VALUE trial. Lancet. 2004;363:2049-51. https://doi.org/10.1016/ S0140-6736(04)16456-8.

6. Martín-Fernández $M$, Vinyoles $E$, Real J, et al. The prognostic value of blood pressure control delay in newly diagnosed hypertensive patients. Hypertension. 2019;37:426-31. https://doi.org/10.1097/HJH.0000000000001896.

7. Egan BM. Prognostic value of blood pressure control delay in newly diagnosed hypertensive patients. J Hypertension. 2019;37:290-1. https://doi.org/10.1097/ HJH.0000000000001944.

8. Simons LA, Chung E, Ortiz M. Long-term persistence with single-pill fixed dose combination therapy versus two pills of amlodipine and perindopril for hypertension: Australian experience. Current Med Res and Opin. 2017;33(10):1783-7. https://doi.org/10.1080/03007995.2017.1367275.

9. Corrao $G$, Parodi A, Nicotra $F$, et al. Better compliance to antihypertensive medications reduces cardiovascular risk. J Hypertension. 2011;29:610-8. https:// doi.org/10.1097/HJH.0b013e328342ca97.

10. Hirakawa $Y$, Arima $H$, Rodgers $A$, et al. Cumulative in-trial and post-trial effects of blood pressure and lipid lowering: systematic review and meta-analysis. Hypertension. 2017;35:905-13. https://doi.org/10.1097/HJH.0000000000001233.

11. Gupta A, Mackay J, Whitehouse A, et al. Long-term mortality after blood pressure-lowering and lipid-lowering treatment in patients with hypertension in the Anglo-Scandinavian Cardiac Outcomes Trial (ASCOT) LEGACY study: 16 years follow-up results of a randomised factorial trial. Lancet. 2018;392:1127-37. https://doi.org/10.1016/S0140-6736(18)31776-8.

12. Dahlöf $B$, Sever PS, Poulter NR, et al. Prevention of cardiovascular events with an antihypertensive regimen of amlodipine adding perindopril as required versus atenolol adding bendroflumethiazide as required in the Anglo-Scandinavian Cardiac Outcomes Trial-Blood Pressure Lowering arm (ASCOT-BPLA): a multicentre randomised controlled trial. Lancet. 2005;366:895-906. https://doi. org/10.1016/S0140-6736(05)67185-1. 